\title{
Linfoma de Burkitt en un escolar con infección perinatal por VIH lentamente progresiva
}

\author{
Claudia Beltrán-Arroyave, Catalina Mesa, Hernán Vásquez, Nayibe Hincapié y Alejandro Díaz-Díaz
}

\section{Burkitt's lymphoma in a school boy with slow progression perinatally acquired HIV}

Children with perinatal human immunodeficiency virus (HIV) infection can present early or late clinical disease. HIV-associated lymphoma is a later manifestation that is associated with advanced immunosuppression (acquired immunodeficiency syndrome -AIDS). This is a case of a 9-year-old boy with recent diagnosis of HIV with Burkitt's lymphoma as first clinical manifestation. In children, the frequency of this association is very low and there are few cases reported.

Key words: Burkitt’s lymphoma; human immunodeficiency virus; children.

Palabras clave: Linfoma de Burkitt; virus de inmunodeficiencia humana; niños.

\section{Introducción}

E 1 cáncer infantil tiene una incidencia mundial de 100 a 150 casos por cada millón de niños y sólo representa $0,5-3 \%$ de las neoplasias malignas en el mundo ${ }^{1}$. Según el reporte de GLOBOCAN, (sobre incidencia, prevalencia y mortalidad estimada de cáncer mundial), en el año 2012 se presentaron 163.284 casos nuevos de cáncer en niños entre los 0 y 14 años, lo que equivale al $1 \%$ del total de casos. Los tumores más frecuentes en la edad pediátrica fueron en primer lugar las leucemias, luego los tumores del sistema nervioso central (SNC) y en tercer lugar los linfomas no Hodgkin ${ }^{2}$.

Según un estudio realizado desde 2009 al 2013 en Cali, Colombia, se observaron 350 casos nuevos de cáncer infantil. Las leucemias tuvieron una incidencia de 44 casos por millón en los menores de 15 años, seguido por los tumores del SNC, con 22 casos por millón y los linfomas con una tasa de incidencia de 15 casos por millón. El linfoma Burkitt fue el menos común de los observados, representando el 3,7\%, con una incidencia de 4 casos por millón ${ }^{3}$.

El linfoma de Burkitt usualmente ocurre en la segunda década de la vida y es poco frecuente en menores de tres años ${ }^{4}$. A diferencia del adulto, los niños típicamente tienen una enfermedad extranodal con afectación del tracto gastrointestinal, mediastino, cabeza y cuello ${ }^{4}$. Las inmunodeficiencias tanto congénitas como adquiridas (VIH o postrasplante) aumentan el riesgo de linfomas no Hodgkin; y también se ha descrito su asociación con el virus de Epstein Barr (VEB) ${ }^{5}$.

Existen diferentes formas de cáncer asociadas a la infección por $\mathrm{VIH}$, especialmente en pacientes que no reciben terapia antirretroviral (TARV) y en estados avanzados de inmunosupresión ${ }^{6}$. Esta asociación es infrecuente en niños ${ }^{7}$, por lo que la evidencia científica sobre su tratamiento es escasa. Adicionalmente, los medicamentos antirretrovirales y quimioterapéuticos tienen importantes interacciones medicamentosas y baja disponibilidad en presentaciones orales adecuadas para este grupo de edad, lo que hace especialmente difícil escoger la terapia más adecuada. Por estas razones, presentamos este caso clínico.

\section{Caso clínico}

Escolar de 9 años, de sexo masculino, con historia de tres semanas de evolución de epistaxis nasal izquierda no asociada a trauma, acompañada de edema, eritema, y dolor en la zona palpebral izquierda. Recibió tratamiento con ampicilina/sulbactam por sospecha de una celulitis periorbitaria preseptal sin presentar mejoría. Se realizó una tomografía computarizada (TC) de senos paranasales que mostró una lesión expansiva obstructiva que comprometía el seno maxilar izquierdo, por lo que fue trasladado para evaluación oncológica. El paciente presentaba pérdida de peso y malestar general de un mes de duración. Tenía antecedente de episodios recurrentes de otitis media supurativa y un episodio de infección del tracto urinario. La madre había fallecido tres años antes, por una causa desconocida al momento de la admisión del niño. El examen físico revelaba buen estado general, sin déficit nutricional. En la hemicara
Universidad de Antioquia. Medellín, Colombia (CBA) Universidad CES. Hospital Pablo Tobón Uribe (CM). Hospital General de Medellín $(H V, N H, A D D)$.

Los autores declaran no tener conflicto de intereses.

Recibido: 23 de marzo de 2017 Aceptado: 22 de agosto de 2017

Correspondencia a: Claudia Beltrán-Arroyave claudia.beltran@udea.edu.co 
izquierda tenía edema de tejidos blandos y protrusión del arco cigomático, con evidencia de una masa en la fosa nasal que la ocupaba en su totalidad y con estigmas de sangrado. Además, tenía adenopatías blandas y móviles ubicadas en la región cervical, submaxilar, axilar izquierda e inguinal bilateral. En el abdomen presentaba una masa dura de aproximadamente ocho centímetros de bordes definidos desde el epigastrio hasta mesogastrio, con extensión hasta el hipocondrio izquierdo. Entre los
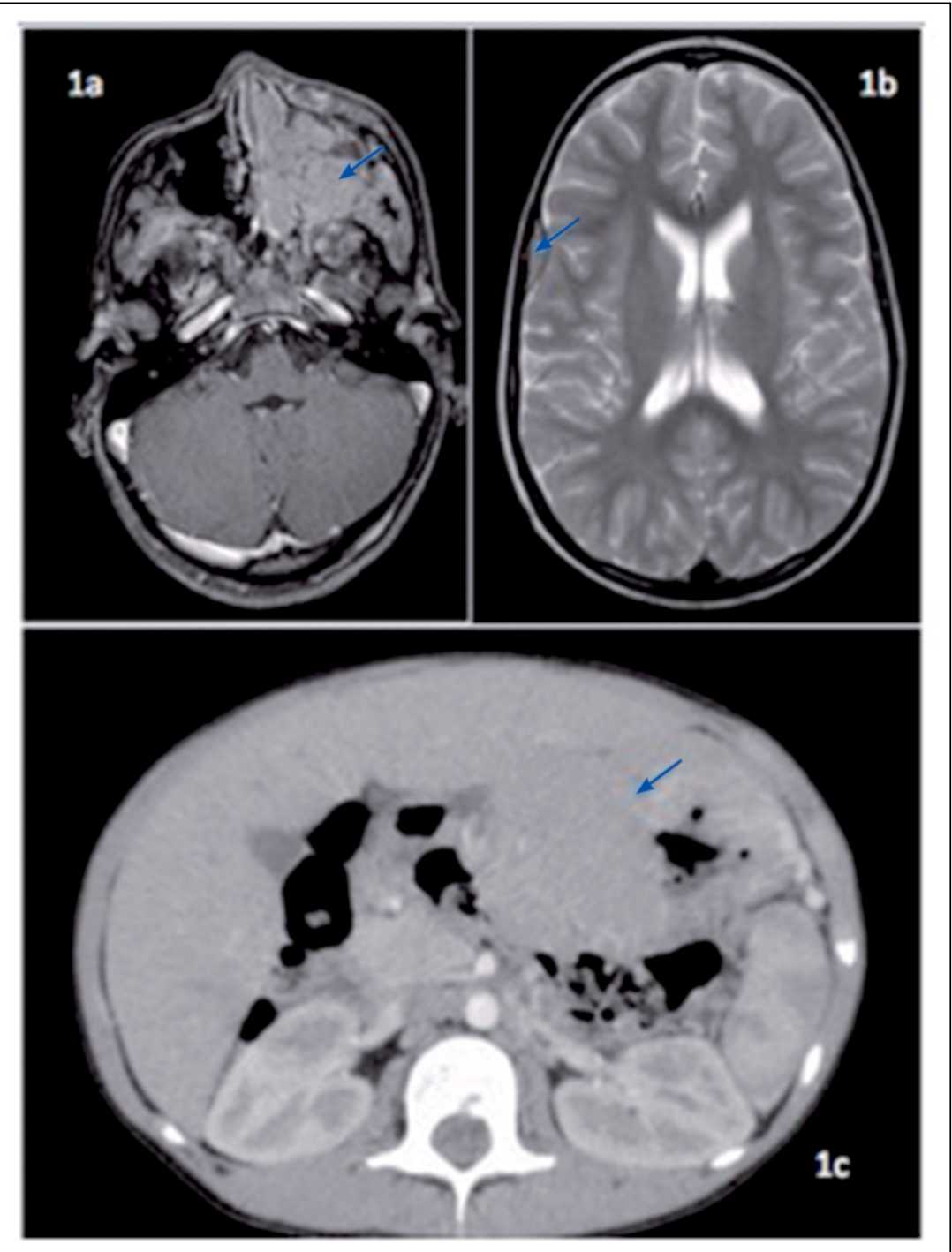

Figura 1. a: RM de senos paranasales: masa de bordes mal definidos con realce heterogéneo que compromete el seno maxilar izquierdo (flecha azul) y las celdillas etmoidales ipsilaterales, disrupción ósea generalizada. Órbitas con disrupción de la tabla interna izquierda; b: RM cerebral: lesión expansiva extra-axial temporoparietal derecha con compromiso leptomeníngeo (flecha azul); c: Lesiones sólidas hipodensas en hígado, localizadas en los segmentos IV y III, la mayor de $10 \mathrm{~mm}$. Masa de 66 × $43 \mathrm{~cm}$ que corresponde a gran engrosamiento de toda la pared gástrica y masas confluentes anteriores al bazo y perigástricas (flecha azul). exámenes de laboratorio presentaba una hemoglobina de $8,2 \mathrm{~g} / \mathrm{dl}$, leucocitos de $5.700 / \mathrm{mm}^{3}$, plaquetas $591.000 /$ $\mathrm{mm}^{3}$, proteína $\mathrm{C}$ reactiva de $14 \mathrm{mg} / \mathrm{dl}$ (VN menor de 1), LDH de 1292 U/L (VN menor de 200). Se realizó una resonancia magnética $(\mathrm{RM})$ de cráneo que confirmó la presencia de una masa maxilar izquierda (Figura 1a) y una lesión temporo-parietal extradural derecha (Figura 1b). La TC de abdomen localizó la masa en el estómago, sin compromiso de otros órganos abdominales. (Figura 1c). La TC de tórax mostró adenopatías axilares y pectorales. La ecocardiografía fue normal. Se realizó una biopsia de la lesión maxilar y extradural. La histopatología e inmunohistoquímica confirmaron una lesión linfoproliferativa de células B compatible con un linfoma no Hodgkin tipo Burkitt. La médula ósea y el LCR fueron negativos para malignidad. Dentro del tamizaje de enfermedades infecciosas (VIH, hepatitis virales A, B y C, CMV, VEB, virus herpes simplex, sífilis, toxoplasmosis) previo al inicio de quimioterapia, la prueba presuntiva para VIH resultó positiva (ELISA de cuarta generación). El diagnóstico se confirmó con una carga viral (ARN RPC) de 169.000 copias $(\log 5,2)$ y un recuento de linfocitos T CD4+ de 81 cél $/ \mathrm{mm}^{3}$ compatible con estadio 3 de la infección. Se realizaron estudios adicionales para buscar infecciones oportunistas (antígeno para Cryptococcus neoformans en sangre y LCR, tuberculina, RPC para M. tuberculosis en tejidos, cultivos para aerobios, hongos y micobacterias en sangre y tejido, serología para hongos (histoplasmosis, paracoccidiomicosis, aspergilosis), los cuales fueron todos negativos. Se inició TARV con raltegravir y tenofovir/emtricitabina para disminuir posibles interacciones medicamentosas con la quimioterapia. Además, recibió profilaxis primaria para Pneumocystis jirovecii y Mycobacterium avium complex (MAC) con cotrimoxazol y azitromicina, respectivamente, además de profilaxis antifúngica con fluconazol durante los episodios de neutropenia secundaria. Una semana después se inició la quimioterapia con el protocolo LNH - B ACHOP $2007^{4}$.

Ambos tratamientos fueron bien tolerados y rápidamente las lesiones comenzaron a disminuir de tamaño. A las cuatro semanas de terapia el niño tenía carga viral indetectable. Finalizó la quimioterapia a los seis meses. Durante el primer año después del diagnóstico y hasta la fecha, la carga viral se ha mantenido indetectable y los linfocitos CD4 se mantienen en niveles sobre 100 cél $/ \mathrm{mm}^{3}$. El paciente no ha presentado complicaciones infecciosas ni no infecciosas relacionadas con el VIH o la quimioterapia. Al re-interrogar a la familia luego de conocer el diagnóstico en el paciente, ellos afirmaron que la mamá había fallecido de SIDA y una infección desconocida. No fue posible tener acceso a los registros médicos de la madre, por lo que no fue posible documentar la forma como se hizo el diagnóstico y la causa exacta de muerte. 


\section{Discusión}

El presente caso ilustra una forma inusual de presentación de la infección por VIH en niños. Las manifestaciones clínicas en los niños con infección por VIH de transmisión perinatal, pueden ser de inicio precoz o tardío; de éstas, las más comunes son las infecciones bacterianas, la neumonía intersticial linfoidea, la hipertrofia parotídea, entre otras, con una edad media de presentación a los tres años $^{8}$. Las enfermedades neoplásicas infantiles asociadas a la infección por VIH son infrecuentes y de presentación tardía. Tienen una incidencia global aproximada del $2,5 \%{ }^{9}$ similar a la reportada por Villarroel y cols. en Chile ${ }^{6}$ y a la reportada por Chen y cols. en Taiwán, con una incidencia de $3 \%{ }^{10}$. En Colombia se encontró únicamente un reporte de un paciente similar al nuestro ${ }^{11}$. Estas son aún más infrecuentes desde que se introdujo la TARV de alta eficacia de forma temprana en el tratamiento del VIH en niños ${ }^{12,13}$. El cáncer infantil es considerado una entidad definitoria del SIDA, y habitualmente ocurre en estadios avanzados de inmunosupresión. Los más frecuentes son los linfomas, principalmente del tipo no Hodgkin y de éstos, el linfoma de Burkitt, que se relaciona además con la infección por $\mathrm{VEB}^{14}$, seguidos del sarcoma de Kaposi y los tumores del músculo liso (leiomiosarcomas) ${ }^{15,16}$.

La mayor susceptibilidad para el desarrollo de cáncer se explica por la activación crónica del sistema inmune inducido por el VIH, así como la participación de virus oncogénicos como el VEB ${ }^{6,17}$. Es así, como el control inmunológico de la enfermedad con el inicio temprano de la TARV de alta eficacia, disminuyó la frecuencia de cáncer significativamente ${ }^{6,18}$. Con una carga viral indetectable se retarda la disfunción del sistema inmune, especialmente de los linfocitos T CD 28 que son fundamentales para la activación de las células $\mathrm{T}^{13,18,19}$.

No existen recomendaciones con alto nivel de evidencia respecto al tratamiento del cáncer asociado al VIH en niños. La recomendación más aceptada, es que, tanto la quimioterapia como la TARV, son igualmente importantes para controlar el cáncer, por eso deben darse simultáneamente ${ }^{20}$. En nuestro paciente se inició la TARV y una semana después la quimioterapia, para vigilar los efectos adversos y evaluar la tolerancia a los medicamentos. Este esquema ha sido recomendado por los expertos para pacientes naive ${ }^{20}$. La única recomendación de no iniciar TARV es en pacientes con mal pronóstico oncológico, que tengan un adecuado recuento de $\mathrm{LT} \mathrm{CD} 4^{21}$.

La elección de la TARV debe ser individualizada. Deben considerarse las interacciones medicamentosas de los fármacos y sus efectos adversos ${ }^{18}$; así como el metabolismo de los ARV especialmente con el sistema citocromo P450 (CYP450) hepático, el cual es compartido con los principales agentes quimioterapéuticos, y cuya función puede verse afectada por estas interacciones y favorecer efectos tóxicos y/o menor respuesta al tratamiento del cáncer o de la infección ${ }^{18}$. Los inhibidores no nucleósidos de la transcriptasa reversa (INNTR), los inhibidores de proteasa (IP), y los inhibidores nucleósidos de la transcriptasa reversa (INTR) son los medicamentos recomendados para iniciar la TARV en niños, pero se deben evitar en pacientes que van a recibir quimioterapia, debido a que los INNTR son inductores y los IP inhibidores de la CYP450 ${ }^{18}$. Además los INTR, principalmente la zidovudina (AZT), producen mielosupresión empeorando la agranulocitosis secundaria a la quimioterapia ${ }^{18}$. Los inhibidores de integrasa, por otro lado, resultan ser la mejor opción terapéutica al no tener estas limitaciones ${ }^{21}$. El antagonista de receptor CCR5 maraviroc, es una de las mejores opciones en pacientes sometidos a trasplante precursores hematopoyéticos (TPH), por su papel en la enfermedad injerto contra hospedero ${ }^{22}$. Por estas razones, el esquema antiretroviral más recomendado en pacientes con cáncer es un inhibidor de la integrasa asociado a una combinación de dos INTR, diferentes a AZT, y evitar el uso de los INNTR y de los IP ${ }^{18}$. Nuestro paciente recibió raltegravir más tenofovir/emtricitabina, basado en estas recomendaciones, con buenos resultados y sin efectos adversos demostrados a la fecha.

La profilaxis para enfermedades infecciosas es similar a la usada en otros pacientes oncológicos o con infección por VIH. En este caso, decidimos iniciar profilaxis primaria con cotrimoxazol y azitromicina para prevenir infecciones por $P$. jirovecii y MAC, respectivamente, debido al bajo recuento de LT CD4 al momento del diagnóstico. En general, debe considerarse el estado serológico previo, el riesgo de exposición a enfermedades transmisibles, el recuento de LT CD4, la duración de la neutropenia, la hipogammaglobulinemia asociada al cáncer y los agentes linfo-tóxicos usados (corticoesteroides a altas dosis) ${ }^{20}$. Se recomienda dar profilaxis con cotrimoxazol para $P$. jirovecii en todos los pacientes, sin importar el recuento de LT CD4, excepto en la fase pre-injerto de los que reciben TPH, por su efecto en la mielo-supresión. La profilaxis con macrólidos para la prevención de MAC se recomienda en pacientes con recuento de LT CD4 menor de 50 cél/ $\mathrm{mm}^{3} \mathrm{o}$ en aquellos en los cuales se anticipa un descenso como efecto de la quimioterapia ${ }^{20}$. La profilaxis antibacteriana, antiviral y antifúngica se determina según el riesgo por el tipo de cáncer y el nivel de inmunosupresión, de acuerdo a lo recomendado en guías específicas ${ }^{20}$. En este caso, no se recomendó profilaxis antibacteriana, pero sí antifúngica durante los episodios de neutropenia febril.

Para el seguimiento de la infección por VIH, no se recomienda el recuento de LT CD4 pues éstos disminuyen por la quimioterapia, sin poder diferenciar si es por efecto inmunológico directo del virus. Se recomienda para la monitorización de la respuesta a la TARV realizar carga viral en forma periódica ${ }^{20}$. 
Es un reto identificar la falla terapéutica a la TARV ${ }^{21}$, especialmente porque los síntomas propios del cáncer y los efectos adversos de la quimioterapia se superponen. Se deben analizar detenidamente las comorbilidades, la evolución virológica, la adherencia, la intolerancia y las interacciones medicamentosas antes de definir un cambio de terapia ${ }^{21}$.

Agradecimientos: A Álvaro Restrepo Pareja, patólogo Hospital General de Medellín.

\section{Resumen}

Las manifestaciones clínicas en los niños con infección por el virus de la inmunodeficiencia humana (VIH) de transmisión perinatal, pueden ser de inicio precoz o tardío. El linfoma asociado a VIH es una manifestación tardía que se asocia a estadios avanzados de inmunosupresión. Se presenta el caso de un escolar de 9 años con diagnóstico de novo de infección por VIH que debutó con un linfoma de Burkitt. En niños, la frecuencia de esta asociación es de $1-2 \%$ con pocos casos reportados en la literatura médica.

\section{Referencias bibliográficas}

1.- Pardo C, Cendales R. Cáncer pediátrico. Incidencia estimada En: Incidencia, mortalidad y prevalencia de cáncer en Colombia, 2007-2011. Primera edición. Bogotá. D.C. Instituto Nacional de Cancerología, 2015; 144. Disponible en: http://www.cancer.gov.co/ files/libros/archivos/incidencia1.pdf (Fecha de acceso: 13 de abril de 2017).

2.- Protocolo de vigilancia en salud pública. Cáncer en menores de 18 años. Instituto nacional de Salud. Bogotá, enero de 2017. Disponible en: http://www.ins.gov.co/ lineas-de-accion/Subdireccion Vigilancia/ sivigila/Protocolos\%20SIVIGILA/PRO $\% 20$ C\%C3\%A1ncer\%20en\%20menores\%20de \%20 18\%20a\%C3\%B1os-.pdf (Fecha de acceso: 18 de agosto de 2017).

3.- Tovar C J R, Gómez G A. Incidencia de cáncer infantil en una ciudad colombiana. Rev Cienc Salud 2016; 14: 315-28.

4.- Vizcaino M, Suárez A, Linares A. Protocolo Nacional LNH-B ACHOP 2007. Protocolo para Linfoma Burkitt o NO Burkitt (Linfomas no Hodgkin de células pequeñas no clivadas indiferenciado Tipo Burkitt o no Burkitt). Colombia, septiembre 15 de 2007. Disponible en: http://achop.org/wp-content/ uploads/2015/03/LNH_ACHOP_2007.pdf (Fecha de acceso: 13 de abril de 2017).

5.- Guía de Práctica Clínica para la detección oportuna, diagnóstico, tratamiento y seguimiento de linfoma de Hodgkin y linfoma no Hodgkin en niños, niñas y adolescentes. Guía No. 10 abril de 2013. Min Salud y Colciencias. P 132. Disponible en: http://www.iets.org.co/ reportes-iets/Documentacin\%20Reportes/ Gu\%C3\%ADa.Completa.Linfomas.2013.pdf
(Fecha de acceso: 13 de abril de 2017).

6.- Villarroel J, Álvarez A M, Chávez A, Cofré J, Galaz M I, Ledesma P, et al. Incidence of cancer in Chilean HIV-infected children. Rev Chilena Infectol 2015; 32: 672-6.

7.- Centers for Disease Control and Prevention. Revised surveillance case definition for HIV Infection-United States, 2014. MMWR Recomm Rep 2014; 63 (RR-3): 1-10.

8.- Beltrán S J, López P, Sierra A. Capítulo: Infección por VIH/SIDA en la edad pediátrica En: Recomendaciones para el abordaje, diagnóstico y tratamiento del niño con VIH/ SIDA. Segunda edición. Comité editorial Catorce. Cali, Colombia. 2011. Pág 19. Disponible en: http://www.slipe.org/pdf/ ManejoNinoVIH-SidaEd2.pdf?idNoticia=103 (Fecha de acceso: 13 de abril de 2017).

9.- Godot C, Patte C, Blanche S, Rohrlich P, Dollfus C, Tabone M D. Characteristics and prognosis of B-cell lymphoma in HIV-infected children in the HAART era. J Pediatr Hematol Oncol 2012; 34: e282-8.

10.- Chen M, Jen I A, Chen Y M. Nationwide study of cancer in HIV-Infected taiwanese children in 1998-2009. J Acquir Immune Defic Syndr 2015; 69: e117-8.

11.- Quiroz L, Vizcaino M, Rengifo L. Linfoma de Burkitt en un niño con infección por VIH. Rev Col Cancer 2004; 8: 36-40.

12.- Chiappini E, Galli L, Tovo P A, Gabiano C, Lisi C, Giaquinto C, et al. Cancer rates after year 2000 significantly decrease in children with perinatal HIV infection: A study by the Italian Register for HIV infection in children. J Clin Oncol 2007; 25: 97-101.

13.- Stebbing J, Gazzard B, Mandalia S, Teague A, Waterson A, Marvin V, et al. Antiretroviral treatment regimens and immune parameters in the prevention of systemic AIDS-related non-Hodgkin's lymphoma. J Clin Oncol 2004; 22: 2177-83.

14.- Simard E P, Shiels M S, Bhatia K, Engels E A. Long-term cancer risk among people diagnosed with AIDS during childhood. Cancer Epidemiol Biomarkers Prev 2012; 21: 148-54.

15.- Launay O, Guillevin L. Epidemiology of HIVassociated malignancies. Bull Cancer 2003; 90: 387-92.

16.- Rees C A, Keating E M, Lukolyo H, Danysh H E, Scheurer M E, Mehta P S, et al. Mapping the epidemiology of Kaposi sarcoma and nonHodgkin lymphoma among children in SubSaharan Africa: a review. Pediatr Blood Cancer 2016; 63: 1325-31.

17.- Werutsky G, de Carvalho G P, da Silva V D, Garicochea B. AIDS-related sinonasal Burkitt lymphoma successfully treated with intensive chemotherapy regimen and high active antiretroviral therapy. Hematol Oncol Stem Cell Ther 2011; 4: 41-4.

18.- Mounier N, Katlama C, Costagliola D, Chichmanian R M, Spano J P. Drug interactions between antineoplastic and antiretroviral therapies: implications and management for clinical practice. Crit Rev Oncol Hematol 2009; 72: 10-20.

19.- Desai S, Landay A. Early immune senescence in HIV disease. Curr HIV/AIDS Rep 2010; 7 : 4-10.

20.- Bower M, Palfreeman A, Alfa-Wali M, Bunker C, Burns F, Churchill D, et al. British HIV Association guidelines for HIV-associated malignancies 2014. HIV Med 2014; 15 Suppl 2: 1-92.

21.- Torres H A, Mulanovich V. Management of HIV infection in patients with cancer receiving chemotherapy. Clin Infect Dis 2014; 59: 106-14. 